\title{
Gastrointestinal pathogens detected by multiplex nucleic acid amplification testing in stools of pediatric patients and patients returning from the tropics
}

\author{
C. Beckmann - U. Heininger • \\ H. Marti · H. H. Hirsch
}

Received: 13 March 2014/ Accepted: 23 June 2014/Published online: 12 July 2014

(c) Springer-Verlag Berlin Heidelberg 2014

\begin{abstract}
Background Gastrointestinal infections are caused by a broad spectrum of pathogens. Conventional diagnostic procedures are resource and time consuming due to single pathogen testing, often in different laboratories.

Method We analyzed 312 consecutive stool samples from pediatric patients $(n=127)$ with gastroenteritis or from adult travelers returning from the tropics with suspected parasite infestation ( $n=185)$ using commercial multiplex nucleic acid amplification testing (NAT) (xTAG gastrointestinal pathogen panel, Luminex) covering 15 diarrheacausing pathogens. The results of the positive samples and a representative number of negative samples were compared to standard methods, including NAT, direct antigen detection (DAD), bacterial culture and microscopy.
\end{abstract}

C. Beckmann · H. H. Hirsch $(\bowtie)$

Division of Infection Diagnostics, Department of Biomedicine

(Haus Petersplatz), University of Basel, Petersplatz 10,

4003 Basel, Switzerland

e-mail: hans.hirsch@unibas.ch

\section{U. Heininger}

Division of Pediatric Infectious Diseases, University Children's Hospital Basel, Basel, Switzerland

H. Marti

Swiss Tropical and Public Health Institute, University of Basel, Basel, Switzerland

H. H. Hirsch

Transplantation and Clinical Virology, Department Biomedicine (Haus Petersplatz), University of Basel, Basel, Switzerland

H. H. Hirsch

Infectious Disease and Hospital Epidemiology, University

Hospital Basel, Basel, Switzerland
Results Of the 185 samples from adult travelers, 21 $(11 \%)$ were multiplexNAT-positive, with enterotoxigenic Escherichia coli (4\%) being the predominant pathogen. Microscopic examination revealed Blastocystis hominis in $23 \%$ not covered by the panel. MultiplexNAT scored positive in 66 pediatric samples (52\%), with rotavirus $(27 \%)$ being the most prevalent. All adenovirus-, rotavirus-, Clostridium difficile- and Cryptosporidium-positive samples were confirmed in external laboratories, but only $40 \%$ of norovirus- and $29 \%$ of Giardia-positive samples. Analysis of frozen specimens by bacterial culture showed the highest discrepancies with the multiplexNAT.

Conclusion Our study demonstrates broad detection of relevant gastroenteritis pathogens by multiplexNAT with a short turnaround time. This is important for diagnosis, infection control and empiric management of gastroenteritis patients, but may be selectively complemented by bacterial culture and resistance testing.

Keywords Gastrointestinal infection - Multiplex PCR · NAT $\cdot$ Stool $\cdot$ Diarrhea $\cdot$ Parasites $\cdot$ Bacteria $\cdot$ Virus

\section{Introduction}

Acute gastroenteritis is a major health problem worldwide. Globally, diarrheal disease was responsible for about 0.75 million fatalities in young children in 2010 [22]. While deaths from diarrhea in industrialized nations are less common, it remains an important cause of morbidity in younger children as well as in adults returning from tropical countries. Accordingly, gastrointestinal diseases result in substantial medical and healthcare expenses, lost productivity, and other costs to society and families [15, 33]. 
Virus, bacteria and parasite infections are typical causes of gastrointestinal disease [40]. The standard methods for detecting these agents are commonly based on microscopy, culture-based identification and direct antigen detection (DAD). However, time-consuming parallel testing for all possible pathogens may be required. A more rapid identification is desirable, especially since a shorter turnaround time could reduce nosocomial transmissions by facilitating infection control measures and specific treatment e.g. for norovirus or Clostridium difficile [9]. Recently, nucleic acid amplification testing (NAT) has been developed for pathogen detection in stool samples, which may permit parallel testing for multiple pathogens by multiplexing and reducing the turnaround time to $<6 \mathrm{~h}$. In this study, we examined the performance of a commercial multiplexNAT called the xTAG GPP (Luminex) using automated nucleic acid extraction and the MAGPIX platform for the detection of viruses, parasites and bacteria in 312 consecutive stool specimens from symptomatic pediatric patients $(n=120)$ and travelers returning from the tropics $(n=176)$.

\section{Materials and methods}

Study design

In total, 312 consecutive stool samples from 296 patients were collected between February 2012 and October 2012. The pediatric group consisted of 120 patients who presented with acute gastroenteritis to the emergency room or were admitted to the University Children's Hospital Basel with suspected viral gastroenteritis. The second group consisted of 151 adult and 25 pediatric patients $(n=176)$ returning from the tropics with gastrointestinal symptoms or parasite infestation submitted by general practitioners from all over Switzerland. The study was approved by the Medical Ethical Committee Basel (EKBB Ref No. 242/12).

Demographics and clinical specimens

The 127 pediatric stool samples were obtained from 120 patients (77 males and 43 females; median age 1 year; interquartile range (IQR) 0 and 2 years; range 6 days to 21 years). The interquartile range is a measure of statistical dispersion being equal to the difference between the upper and the lower quartiles IQR $=\mathrm{Q} 3-\mathrm{Q} 1$. The 185 stool samples from travelers were obtained from 176 patients (78 males, 98 females; median age 40; IQR 28 and 53 years; range 2-78 years; Table 1). All samples were aliquoted and frozen at $-80{ }^{\circ} \mathrm{C}$ and then analyzed by multiplexNAT. All multiplexNAT-positive samples and a random selection of 72 multiplexNAT-negative samples were sent blinded to external laboratories for independent analysis.
Table 1 Demographic characteristics of the patients

\begin{tabular}{ll}
\hline Parameter & No. of patients (\%) \\
\hline All patients & 296 \\
Gender, male/female & $155(52) / 141(48)$ \\
Age range & $0-78$ \\
Pediatric patients & 120 \\
Gender, male/female & $77(64) / 43(36)$ \\
Age & \\
$0-1$ & 47 \\
$1-5$ & 54 \\
$5-21$ & 19 \\
Patients returning from tropics & 176 \\
Gender, male/female & $78(44) / 98(56)$ \\
Age & \\
$0-1$ & 0 \\
$1-5$ & 5 \\
$5-21$ & 20 \\
$21-65$ & 133 \\
$>65$ & 18 \\
\hline
\end{tabular}

Sample pre-treatment and nucleic acid extraction

Native stool samples $(50 \mathrm{mg})$ were transferred from sterile containers to Bertin SK38 soil kit bead tubes (Bertin Technologies, Montigny, France), which contain a mix of glass and ceramic beads in $2 \mathrm{ml}$ standard tubes. After adding $1 \mathrm{ml}$ of NucliSENS easyMAG lysis buffer (bioMérieux, Genève, Switzerland) and $10 \mu \mathrm{l}$ of Escherichia coli phage MS2 (Luminex Molecular Diagnostics Inc., Toronto, Canada) to monitor the efficiency of nucleic acid extraction and amplification, the tubes were vortexed for $5 \mathrm{~min}$, incubated at room temperature for $10 \mathrm{~min}$ and then centrifuged at $14,000 \mathrm{rpm}$ for $2 \mathrm{~min}$ to pellet insoluble material. $500 \mu \mathrm{l}$ of the supernatant was transferred to the extraction tubes (Abbott Molecular, Baar, Switzerland).

Total nucleic acids were extracted from $200 \mu \mathrm{l}$ of supernatant using the Abbott $m 2000_{\text {sp }}$ instrument (Abbott Molecular, Baar, Switzerland) and the Sample Preparation System $_{\text {DNA }}$ Kit according to the Open-Mode-Protocol for DNA/RNA (RNADNA-Plasma-LL-200-70) of the manufacturer's instructions. The DNA/RNA extract was eluted into $70 \mu \mathrm{l}$ of $m$ Elution Buffer ${ }_{\text {DNA }}$, of which $10 \mu \mathrm{l}$ was used immediately for the multiplex RT-PCR. The remaining extracts were stored at $-80{ }^{\circ} \mathrm{C}$ until further use.

\section{MultiplexNAT}

Total nucleic acids extracted from fecal samples were analyzed by multiplexNAT using the xTAG Gastrointestinal Pathogen Panel assay (Luminex Molecular Diagnostics Inc., Toronto, Canada) according to the manufacturer's 
instructions. The multiplexNAT simultaneously detects 15 pathogens consisting of Salmonella sp., Shigella sp., Shiga toxin-producing E. coli (STEC) stx1/stx2, Vibrio cholerae, Yersinia enterocolitica, $C$. difficile toxin A/B, Campylobacter sp., E. coli O157, Enterotoxigenic E. coli (ETEC) LT/ST, adenovirus 40/41, rotavirus A, norovirus GI/GII, Giardia lamblia, Cryptosporidium sp. and Entamoeba histolytica. Technically, a multiplex reverse transcriptase PCR is done using target-specific tagged primers and biotinylated primers. Amplification products are detected by hybridization to the pathogen-specific complementary antitag sequence coupled to specific beads and binding of the streptavidin-phycoerythrin reporter to the biotinylated primers. After washing steps, the resulting median fluorescence intensities (MFI) were analyzed by the Luminex MAGPIX instrument and TDAS software version 1.0. An MFI value above the threshold for a particular pathogen and a $\mathrm{S} / \mathrm{N}$ ratio above 10 indicated a positive result. The limit of detection (LOD) was evaluated by the manufacturer for each pathogen and can be taken from the instruction manual. Intra-assay reproducibility was assessed using 11 stool samples analyzed on two different days and revealed $100 \%$ concordance for all samples.

\section{Direct antigen detection (DAD)}

Direct antigen detection for rotavirus, adenovirus and norovirus was performed using the Diarlex Rota-Adeno latex test (Orion Diagnostica Oy, Espoo, Finland) and the RIDA $^{\circledR}$ QUICK norovirus immunochromatographic lateral flow test (r-biopharm, Darmstadt, Germany), respectively. In brief, for the adeno-rotavirus DAD, a 1:10 dilution of stool specimens in $0.05 \mathrm{M}$ Tris buffer $\mathrm{pH} 7.2$ was vortexed at room temperature for $2 \mathrm{~min}$, and then centrifuged for $10 \mathrm{~min}$ at $1,000 \times g$. Approximately $50 \mu \mathrm{l}$ of the supernatant was gently mixed with an equal volume of the test latex reagent on a test card. The result was positive if agglutination occurred within 2 min and no agglutination was observed with the control latex reagent. For the norovirus DAD, $0.5 \mathrm{ml}$ of reagent $\mathrm{A}$ and $0.5 \mathrm{ml}$ of reagent B was mixed in a reaction vessel. Approximately $50 \mu \mathrm{l}$ of liquid stool or $50 \mathrm{mg}$ of solid stool sample was added to the reagents to yield a suspension. The suspension was incubated at RT for $5 \mathrm{~min}$. Of the particle-free supernatant, $150 \mu \mathrm{l}$ was placed into the test cassette. DAD was positive, if the indicator line and the control line appeared within $15 \mathrm{~min}$.

\section{Parasite detection}

All samples from patients returning from the tropics were examined directly by microscopy after SAF concentration at a magnification of $500 \times$ for protozoa and a magnification of $100 \times$ for helminths [24]. For the detection of Cryptosporidium sp., a smear was read after SAF concentration and staining with the modified Ziehl-Neelson stain [16].

\section{Confirmatory testing}

Frozen aliquots of the fecal samples positive for one or multiple pathogens were sent to different external laboratories on dry ice. Samples went through two freeze-thaw cycles before analysis in the reference laboratory. The samples were analyzed either by nucleic acid extraction, followed by a validated PCR or by culture on selective media for enrichment and identification either with or without previous priming in adequate enrichment broths. The $C$. difficile diagnostics included simultaneous detection of enterotoxin A/cytotoxin B and glutamate dehydrogenase (GLDH) antigen using the C. diff Quick Chek Complete kit (Alere GmBH, Kölm, Germany), and a DNA strip based technology (Hain Lifescience GmBH, Nehren, Germany) that allows differentiation of $C$. difficile, toxin A (tcdA) and $\mathrm{B}$ (tcdB) genes, binary toxin, deletions in regulatory gene tcdC, as well as detection of moxifloxacin resistance. External laboratories were the National Reference Center for Enteropathogenic Bacteria and Listeria (NENT) at the University of Zürich in Zürich, Switzerland; the Bernhard Nocht Institute for Tropical Medicine in Hamburg, Germany; the Consulting Laboratory for Rotaviruses and Noroviruses at the Robert-Koch-Institute in Berlin, Germany; the National Reference Laboratory for Adenoviruses at the Institute of Virology of the Hannover Medical School in Hannover, Germany; and the Consulting Laboratory for $C$. difficile at the Institute for Medical Microbiology and Hygiene, University of the Saarland in Homburg/Saar, Germany. As negative controls, 72 random samples not positive in the multiplexNAT analysis were also sent to the reference laboratories.

MultiplexNAT-positive stool samples detecting Shigella sp., Salmonella sp., Campylobacter, Shiga toxin-producing E. coli (STEC) stx $1 / \mathrm{stx} 2$, or enterotoxigenic E. coli (ETEC) were thawed and cultured on selective media at the National Reference Center for Enteropathogenic Bacteria and Listeria using standard clinical bacteriology [30]. In brief, for detection of Campylobacter sp, a fecal suspension was cultured on Brilliance CampyCount agar (Oxoid, Wesel, Germany) for $48 \mathrm{~h}$ at $41.5^{\circ} \mathrm{C}$ in a microaerophilic atmosphere. For culture of Salmonella sp., fecal suspension was grown on MacConkey agar (Oxoid) and after selenite and Rappaport-Vassiliadis enrichment (Oxoid), on xylose lysine deoxycholate (XLD) (Oxoid) and mannitol lysine crystal violet brilliant green (MLCB) (Oxoid) agar plates. Salmonellae grow as large purple-black colonies due to hydrogen sulfide production after $18-24 \mathrm{~h}$ at $36^{\circ} \mathrm{C}$. 
Targets of the commercial multiplex PCR included the genes for stx 1 and stx 2 from STEC; the adhesin intimin, responsible for the intimate attachment to the enterocyte membrane and the formation of attaching and effacing (A/ E) lesions that is encoded by the eaeA gene, present in EPEC; the genes for heat-labile (LT) and heat-stable (ST) enterotoxins of ETEC [31]; and finally the ipaH gene, the invasion plasmid antigen $\mathrm{H}$ that is used for detecting Shigella and EIEC. In addition, the virulence-associated regulatory genes $\operatorname{aggR}$ and $\operatorname{agg} 3 \mathrm{C}$ as well as the virulenceassociated plasmid pCVD432 of the EAEC [29], were targeted in a specific single PCR. The tests were combined with a commercial multiplex PCR Primer Mix DEC (Diarrhea-causing E. coli) (Statens Serum Institute, Hillerød, Denmark) specific for stx1, stx2, eaeA, LT, ST and $i p a H$ to detect STEC, enteropathogenic E. coli (EPEC), enterotoxigenic E. coli (ETEC), Shigella and enteroinvasive E. coli (EIEC), respectively. Furthermore, enteroaggregative E. coli (EAEC) were examined by $\operatorname{aggR}$ - [5], pCVD432- [36] and agg3C-specific [3] single PCR analyses. All PCR analyses were performed on colonies grown on MacConkey agar after 24- and 48-h incubation.

\section{Statistics}

Sensitivity, specificity, positive predictive value (PPV) and negative predictive value (NPV) were calculated for the mulitplexNAT in comparison to DAD, real-time PCR and bacterial culture using $2 \times 2$ tables. Agreement between multiplexNAT, conventional methods and realtime PCR was assessed by the kappa value test. Kappa values from 0.21 to 0.4 represent fair agreement; from 0.41 to 0.6 moderate agreement; 0.61 to 0.8 and 0.81 to 0.99 indicate substantial and almost perfect agreement, respectively.

\section{Results}

Of 312 samples, 87 (28\%) were positive by multiplexNAT for at least one pathogen. Single infections were most prevalent as co-infections were found in only eight samples (3\%; children, $4 \%$; travelers, $1.6 \%$ ). For the 127 samples obtained from the 120 pediatric patients, multiplexNAT was positive in 66 (52\%) fecal samples. Thirteen stool samples were tested from six patients reflecting 11 different episodes, of which only one yielded a discordant result for norovirus detection. For the 185 samples obtained from the 176 travelers returning from the tropics, multiplexNAT was positive in $21(11 \%)$. The different rates in the two study groups suggested differences between the patient populations such as age and exposure risks.
Pediatric patient samples

The pediatric patients either presented to the emergency room or had been admitted to hospital, and 101 were younger than 5 years of age (84\%; median age 1 year; IQR 0 and 1 year; range 6 days to 4 years; Table 1 ). As a viral gastroenteritis was suspected on clinical grounds, the fecal samples were initially analyzed for rotavirus and adenovirus by DAD. The multiplexNAT panel detected nine pathogens including rotavirus, norovirus, $C$. difficile, Salmonella sp., adenovirus, Campylobacter sp., G. lamblia, ETEC, and Cryptosporidium sp. (Fig. 1a). Not detected by multiplexNAT were $V$. cholerae, Y. enterocolitica, E. histolytica, E. coli STEC, Shigella sp., and E. coli O157. The seasonal distribution during the 8 months of collection showed a relative predominance of viral pathogens during cold season, and bacterial pathogens during the warm season (Fig. 2).

In the 34 samples found positive for rotavirus by multiplexNAT, $23(68 \%)$ samples were also positive for rotavirus by DAD (Fig. 3). Nine (26\%) of 34 rotaviruspositive samples were obtained from children $<1$ year of age, 21 (62\%) from children between 1 and 2 years of age. Adenovirus was found by multiplexNAT in four samples, which were also detected by DAD (100\%). They originated in three cases from children $<2$ years of age, and from one 12-year old child. Of the 11 positive norovirus samples, only seven had enough material left to be tested by DAD being positive in three (43\%) specimens (Fig. 3). Using DAD as reference, the multiplexNAT had a sensitivity of $100 \%$ for rotavirus and adenovirus, and a specificity of $100 \%$ for adenovirus, and $89.4 \%$ for rotavirus. For norovirus, the multiplexNAT had a sensitivity of $100 \%$ and a specificity of $96.8 \%$. No correlation was seen between level of MFI obtained in the multiplexNAT assay and detection by the antigen test for rotavirus and norovirus. DAD was used for pediatric samples only, since the primary indication for testing samples from patients returning from the tropics was the search for parasitic disease.

Pathogens in samples of patients returning from the tropics

The 176 mostly adult travelers consisted of 78 males and 98 females, including 25 children (median age 40; IQR 28 and 53 years; range 2-78 years). In $11 \%$, multiplexNAT detected eight different pathogens including ETEC, $G$. lamblia, norovirus, Shigella sp., and Campylobacter sp., Salmonella sp., adenovirus and C. difficile (Fig. 1b), whereas rotavirus, Shiga toxin-producing E. coli (STEC), E. coli O157, V. cholerae, Y. enterocolitica, E. histolytica and Cryptosporidium sp. were not detected. 
Fig. 1 Distribution of pathogens in pediatric samples and samples from travelers returning from the tropics detected by multiplexNAT. a Distribution of pathogens in 127 pediatric samples collected from 120 children $(5$ coinfections). b Distribution of pathogens in 185 fecal samples collected from 176 travelers returning from the tropics ( 3 coinfections)
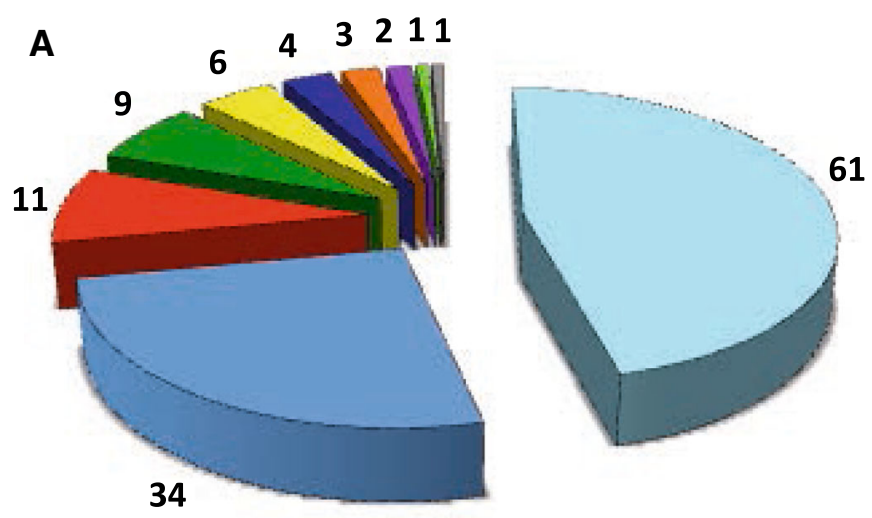

$61 \square$ Norovirus (9\%)

口 C. difficile (7\%)

$\square$ S. enterica (5\%)

- Adenovirus (3\%)

口 Campylobacter (2\%)

Giardia (2\%)

$\square \operatorname{ETEC}(0.8 \%)$

口 Cryptosporidium (0.8\%)

B

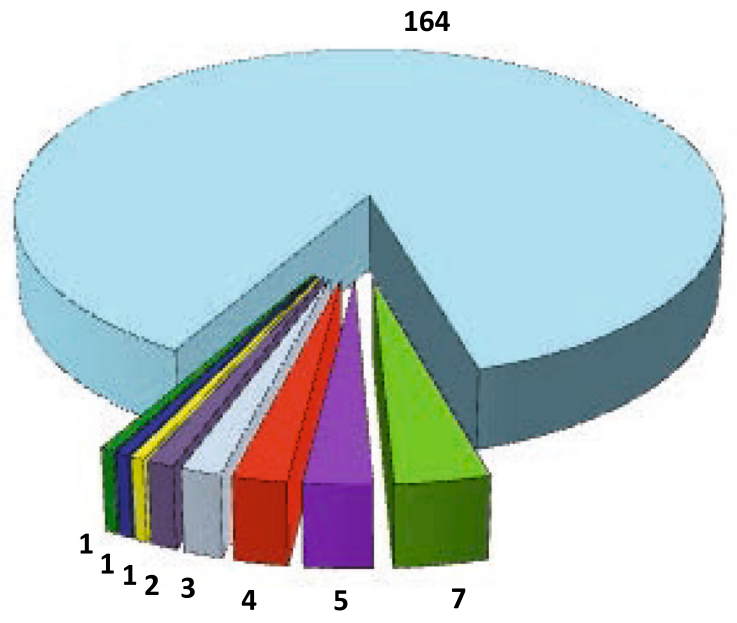

\section{$\mathbf{N}=185$ samples}

$\square$ negative (89\%)

$\square \operatorname{ETEC}(4 \%)$

口 Giardia (3\%)

口 Norovirus (2\%)

口 Shigella (2\%)

口 Campylobacter (1\%)

$\square$ S. enterica (0.5\%)

- Adenovirus $(0.5 \%)$

口 C. difficile $(0.5 \%)$
Fig. 2 Temporal distribution of infections in pediatric samples

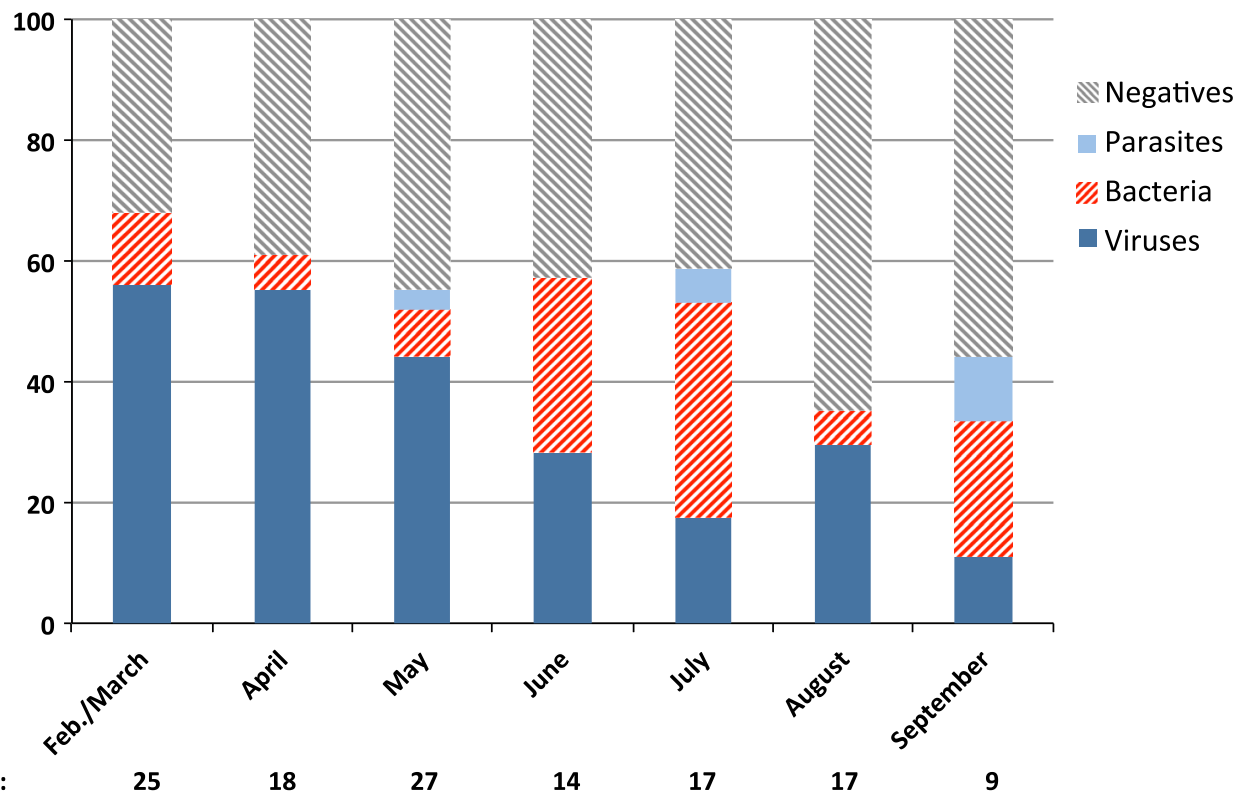


Fig. 3 Comparing MultiplexNAT with virus DAD and PCR Detection

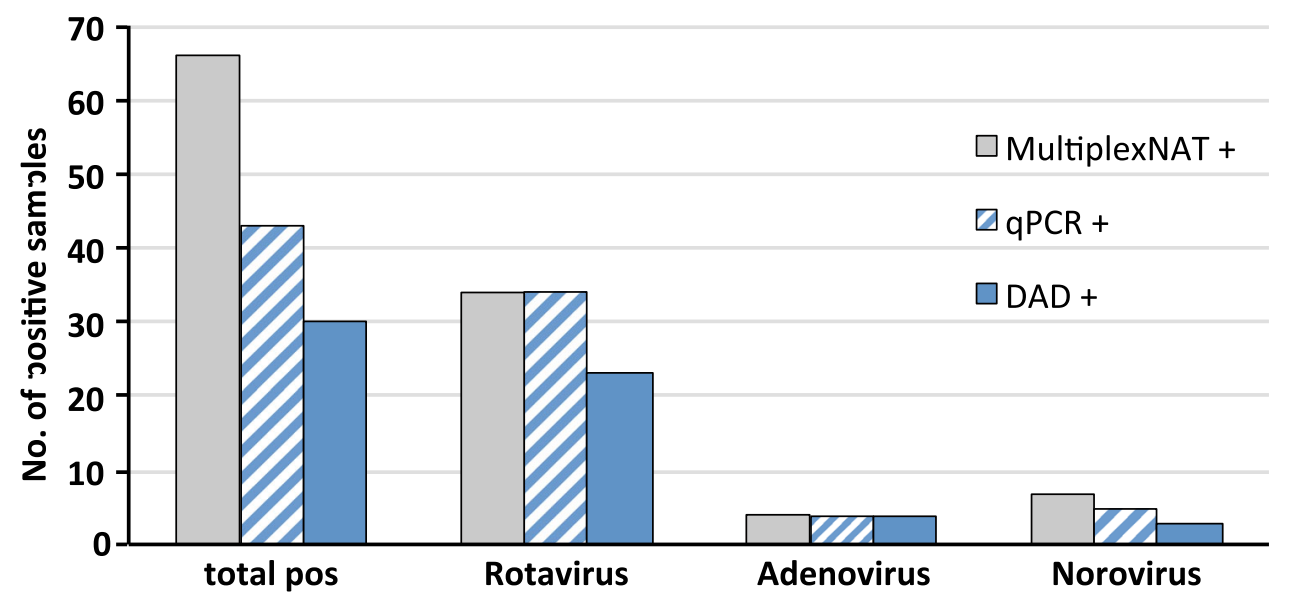

Table 2 Overview of microscopic results in comparison to multiplexNAT

\begin{tabular}{|c|c|c|}
\hline Pathogen & $\begin{array}{l}\text { No. of positives using } \\
\text { microscopy }(\%)\end{array}$ & $\begin{array}{l}\text { No. of positives using } \\
\text { multiplexNAT }(\%)\end{array}$ \\
\hline Protozoans & 63 (34) & $5(3)$ \\
\hline $\begin{array}{l}\text { Blastocystis } \\
\text { hominis }\end{array}$ & $42(23)$ & \\
\hline Endolimax nana & $14(8)$ & \\
\hline $\begin{array}{l}\text { Entamoeba } \\
\text { hartmanni/coli }\end{array}$ & $13(7)$ & \\
\hline $\begin{array}{l}\text { Entamoeba } \\
\text { histolytical } \\
\text { dispar }\end{array}$ & $9(5)$ & 0 \\
\hline $\begin{array}{l}\text { Jodamoeba } \\
\text { bütschlii }\end{array}$ & $3(2)$ & \\
\hline $\begin{array}{l}\text { Chilomastix } \\
\text { mesnili }\end{array}$ & $2(1)$ & \\
\hline Giardia lamblia & $1(0.5)$ & $5(3)$ \\
\hline Cyclospora & $1(0.5)$ & \\
\hline Microsporidia & 0 & \\
\hline Cryptosporidia & 0 & 0 \\
\hline Helminthes & $3(2)$ & \\
\hline $\begin{array}{l}\text { Strongyloides } \\
\text { stercoralis }\end{array}$ & $1(0.5)$ & \\
\hline $\begin{array}{l}\text { Ascaris } \\
\text { lumbricoides }\end{array}$ & $1(0.5)$ & \\
\hline $\begin{array}{l}\text { Hookworm } \\
\text { (eggs) }\end{array}$ & $1(0.5)$ & \\
\hline Dual infections & $12(6)$ & \\
\hline Triple infections & $5(3)$ & \\
\hline
\end{tabular}

The 185 fecal samples were initially examined by microscopy for protozoa and helminths infection (Table 2). In 63 samples (34\%) a positive result was reported, with Blastocystis hominis being the most frequently detected (42/63; $67 \%)$, followed by Endolimax nana (14/63; 22 \%), Entamoeba hartmanni/coli (14/63; $22 \%)$ and E. histolytica/dispar (9/63; $5 \%)$. Microscopy revealed $12(6 \%)$ double and 5 (3\%) triple infections; the majority of them involved $B$. hominis $(12 / 17 ; 71 \%)$.

To compare the results of the multiplexNAT with standard microscopy, only data for the three parasites included in the panel (G. lamblia, E. histolytica and Cryptosporidium sp.) could be taken into account (Table 2). Absence of Cryptosporidium sp. was confirmed by multiplexNAT, however, four additional cases of $G$. lamblia infection were revealed $(n=5 ; 2.7 \%)$. E. histolytica was not detected by the mulitplexNAT, and the microscopy-positive results were identified as Entamoeba dispar, coli and hartmanni.

After introduction of the multiplexNAT assay in our routine diagnostics, 69 consecutive samples from 43 patients with diarrhea (24 males and 19 females, median age, 55 years; IQR 44 and 65 years; range 24-79 years) were analyzed. A positivity rate of $29 \%$ was revealed, with the leading pathogens being norovirus (10\%), $C$. difficile (4\%) and Campylobacter sp. (4\%). Thus, the distribution was similar to the one found in the pediatric gastroenteritis patients.

Comparison of the multiplexNAT with conventional diagnostics

Samples testing positive by multiplexNAT were sent off for independent blinded testing in different external laboratories. For eight samples, sufficient material was not available. For the remaining 64, 53 (83\%) were confirmed. All samples that were sent in for analysis of rotavirus, adenovirus, Cryptosporidium sp. and $C$. difficile were confirmed giving rise to $100 \%$ sensitivity, specificity, PPV and NPV and a perfect interobserver agreement of $\kappa=1$ (Table 3). Additional sequencing of the adenoviral DNA revealed adenovirus group $\mathrm{F}$, serotype 41 for all adenovirus-positive samples. Adenovirus group F, serotypes 40 
Table 3 Comparing multiplexNAT with PCR testing

\begin{tabular}{llrrrl}
\hline & $\begin{array}{l}\text { Sensitivity } \\
(\%)\end{array}$ & $\begin{array}{l}\text { Specificity } \\
(\%)\end{array}$ & $\begin{array}{l}\text { PPV } \\
(\%)\end{array}$ & $\begin{array}{l}\text { NPV } \\
(\%)\end{array}$ & $\kappa$ \\
\hline Rotavirus & 100 & 100 & 100 & 100 & 1 \\
Adenovirus & 100 & 100 & 100 & 100 & 1 \\
Norovirus & 100 & 42 & 46 & 100 & 0.32 \\
C. difficile & 100 & 100 & 100 & 100 & 1 \\
Salmonella sp. & 100 & 91 & 43 & 100 & 0.6 \\
Cryptosporidium & 100 & 100 & 100 & 100 & 1 \\
G. lamblia & 100 & 56 & 33 & 100 & 0.32 \\
\hline
\end{tabular}

$P P V$ positive predictive value

$N P V$ negative predictive value

$\kappa$ interobserver agreement

and 41 have been most commonly associated with acute gastroenteritis in children and adults [38]. Of the 15 multiplexNAT norovirus-positive samples, only six (40\%) could be confirmed by real-time PCR. MultiplexNAT revealed that six of the norovirus-positive samples from children belonged to genogroup II (GII), three samples to genogroup I (GI), and two to each genogroup of the four positive diarrheal samples from patients returning from the tropics. Two specimens could not be repeated due to lack of material. Sensitivity for norovirus was calculated to be $100 \%$, specificity $42 \%$, the PPV was $46 \%$ with a fair agreement of $\kappa=0.32$. Seven specimens were positive for G. lamblia by multiplexNAT. Of these, only two tested positive by real-time PCR (29\%). The MFI values of the non-confirmed samples ranged from 226 to 878, suggesting a lower parasitic load. The MFI values of the two positives specimens were at 1,428 and 1,561, of which the latter was microscopically positive. Due to lack of specimen, one of the samples could not be tested by real-time PCR. Sensitivity for G. lamblia was $100 \%$, specificity $56 \%$, the PPV was calculated to be only $33 \%$; a fair agreement between real-time PCR and the multiplexNAT assay resulted in $\kappa$ of 0.32 (Table 3).

All $C$. difficile-positive samples were confirmed by DNA-hybridization for $C$. difficile and the toxin A and B genes, as well as in the $C$. difficile antigen (GLDH) assay. The cytotoxin $\mathrm{AB}$ test was positive in six out of nine samples, culture in only three samples. Two of the culture-positive samples belonged to PCR ribotype 14/66, one to PCR ribotype 10 . Other bacterial pathogens were detected by multiplexNAT in 10 stool samples from children $(8 \%)$ and 11 samples from travelers returning from the tropics $(6 \%)$. These 21 positive specimens were found positive for ETEC LT/ST $(n=8)$, Campylobacter sp. $(n=5), S$. enterica $(n=7)$ and Shigella sp. $(n=3)$ and were re-analyzed by either bacterial
Table 4 Co-infections in all samples analyzed by mulitplexNAT

\begin{tabular}{llll}
\hline $\begin{array}{l}\text { Age (month/ } \\
\text { year) }\end{array}$ & $\begin{array}{l}\text { Gender male/ } \\
\text { female }\end{array}$ & Pathogen 1 & Pathogen 2 \\
\hline $\begin{array}{l}\text { Pediatric patients } \\
2 \text { months }\end{array}$ & $\mathrm{M}$ & Norovirus & Salmonella sp. \\
6 months & $\mathrm{M}$ & Rotavirus & Norovirus \\
10 months & $\mathrm{M}$ & Rotavirus & C. difficile \\
2 years & $\mathrm{M}$ & Rotavirus & C. difficile \\
2 years & $\mathrm{W}$ & Rotavirus & Giardia lamblia \\
Patients returning from tropics & & \\
48 years & $\mathrm{M}$ & ETEC LT/ & Shigella sp. \\
& & ST & \\
65 years & $\mathrm{W}$ & ETEC LT/ & Giardia lamblia \\
& & ST & \\
74 years & $\mathrm{W}$ & ETEC LT/ & Campylobacter \\
& & ST & sp. \\
\hline
\end{tabular}

culture (for Salmonella sp. and Campylobacter sp.) or by PCR (for STEC, EPEC, ETEC, EIEC/Shigella, and EAEC).

Culture of frozen samples for non-spore-forming bacterial pathogens and multiplexNAT showed the greatest discrepancies. Three of seven Salmonella sp. positives (sensitivity $100 \%$; specificity $91 \%$; PPV $43 \%$; $\kappa$ 0.6; Table 3) and none of the ETEC-, Campylobacter- and Shigella-positive samples could be confirmed $(n=17)$. The majority of samples did not yield bacterial growth on enrichment or selective media $(n=11)$; seven samples were negative by culture and specific PCR (ETEC, Salmonella sp. and Shigella sp.) for the respective pathogen. The five specimens positive for Campylobacter sp. by multiplexNAT could not be recovered on selective medium. However, two of three positive multiplexNAT results from pediatric samples were documented as positive cultures in the patient records. No pathogen was found in any of the 72 negative control samples.

\section{Co-infections by multiplexNAT}

Co-infections were detected in five children and in three travelers (Table 4). The majority of the multiple infections included rotavirus $(n=4)$, followed by ETEC LT/ST $(n=3)$, C. difficile $(n=2)$, norovirus $(n=2)$ and Giardia $(n=2)$. Salmonella sp., Shigella sp. and Campylobacter sp. were part of mixed infections one time each. Discordant samples were re-extracted and analyzed for a second time in the multiplexNAT assay, starting from the frozen material. In total, 5/8 mixed infections found in the first round of analysis by multiplexNAT could be confirmed by $\mathrm{PCR}$, or a repeat multiplexNAT. 


\section{Discussion}

In this study, we examined the performance of a commercial multiplexNAT detecting 15 gastroenteritis pathogens in 312 consecutive stool samples from two different patient groups. Overall, pathogens were identified in 87 stool samples (28\%), 66 of which were obtained from pediatric patients with gastroenteritis. This detection rate of $52 \%$ was significantly higher than the $21(11 \%)$ in samples from mostly adult patients with suspected parasite infestation ( $p<0.0001$; Chi square). In the pediatric samples, the pathogen distribution corresponds to the one expected for diarrhea in young children [2, 6, 34, 37].

Rotavirus was the most frequently detected pathogen in pediatric samples $(27 \% ; 34 / 127$ or $52 \%$ of positive samples), and also most prevalent in co-infections (4/5). Rotavirus vaccination is not yet part of the standard vaccine program in Switzerland, explaining the high prevalence of this virus in young children. The multiplexNAT increased detection rates by $32 \%$ compared to DAD and validity of the data was confirmed by independent rotavirus-specific PCR, resulting in a PPV of $100 \%$ and $\kappa=1$. With regard to co-infections, the data are in accordance with other studies [2, 6, 34], where rotavirus was found prominently in young children with acute gastroenteritis. Possibly, rotavirus might play a role in conjunction with primary bacterial or facilitating secondary bacterial infections [35].

MultiplexNAT also reliably detected other pathogens i.e. adenovirus, Cryptosporidium sp., $C$. difficile), yielding a PPV of $100 \%$, and an interobserver agreement of $\kappa=1$. Interestingly, $C$. difficile was detected in $7 \%$ of children's samples. C. difficile associated disease is one of the main causes of nosocomial gastroenteritis [1] and is also linked to antibiotic-associated diarrhea [18]. However, in a recent study by Denno et al. [11], the role of $C$. difficile as causative agent has been questioned. Despite the increasing epidemiological importance of community-acquired $C$. difficile colitis and the fact that the current molecular assays focus on toxin gene detection, it remains to be addressed whether or not children are a relevant community reservoir of $C$. difficile, and if its detection always warrants treatment.

MultiplexNAT detected a total of eight ETEC-positive samples, five Campylobacter-positive samples, seven $S$. enterica-positive samples, and three Shigella-positive samples in this study. While culture methods confirmed three of the seven Salmonella-positive samples (43\%), they failed to confirm any of the Campylobacter and Shigella results. Of the unconfirmed Salmonella cases, three showed clinical signs of enteritis and fever. Unfortunately, for one patient, clinical records were not available. Similarly, the eight multiplexNAT ETEC results could not be independently found by PCR in an external referral laboratory, since primary enrichment by culture was not successful after thawing. Therefore, sensitivity, specificity, PPV and NPV could not be calculated reliably for these bacteria. False-positive results in the multiplexNAT or, more likely to us given the technical limitation, falsenegative results in the external lab might explain this discordance. Shigella-positive samples were all confirmed by independent repeats of the mulitplexNAT. Since samples that were sent in for bacterial examination were frozen at $-80{ }^{\circ} \mathrm{C}$, most likely viability was greatly reduced, thereby contributing to discordant analyses. The positive findings of some culture-based original patient records described in the results section for two of three Campylobacter sp. positive pediatric samples, also support this conclusion. For Campylobacter sp., it has been reported that freezing [28] and the age of the culture [32] greatly influence the survival of non-spore-forming bacteria.

Norovirus was present in 11/66 (15\%) pathogen-positive children's specimens and in 4/21 (17\%) positive traveler's samples being a most prevalent pathogen in both patient groups. Incidence rates in children range from 7 to $20 \%[13,19,21,23]$. Detection rates of norovirus by multiplexNAT were considerably higher $(>50 \%)$ than by DAD, in line with the well-known lower sensitivity of DAD assays. However, the PPV for norovirus was only $46 \%$, with a fair $\kappa$ value of 0.32 , when compared with an external reference PCR. Norovirus-positive specimens missed by external PCR testing had a wide range of MFI values in the multiplexNAT suggesting that low viral loads are less likely causing the discrepancies. Therefore, the high sequence variability of noroviruses is a contributing cause of reduced sensitivity. Sequencing of the corresponding norovirus target regions in the discordant samples might clarify this possibility.

The most common cause of traveler's diarrhea is enterotoxigenic E. coli (30-60\% of all cases) [4, 17]. We detected ETEC in $33 \%(7 / 21)$ of the positive stool samples. The second most frequently detected pathogen was the parasite $G$. lamblia, which was detected in 5/21 positive samples $(21 \%)$ and in $3 \%$ of samples overall. The prevalence in travelers is between 3 and $4 \%$ for Switzerland (Swiss Tropical and Public Health Institute, Basel, Switzerland). Compared with the multiplexNAT, microscopy, only one Giardia-positive sample was found by microscopy, similar to other reports [7, 39].

No association was seen between Cryptosporidium infection and travel history. However, cryptosporidiosis can be linked to public swimming pools and lake water [20] and young children are known to be particularly susceptible [8, 25, 26]. In our study, the only confirmed infection occurred in a pediatric sample from an 8-year-old girl with no known travel history. 
For the mostly adult patients returning from the tropics with gastrointestinal symptoms or parasite infestation, the value of this mulitplexNAT is not as clear as for the pediatric gastroenteritis group. Microscopy identified 63 positive samples (34\%), whereas multiplexNAT revealed pathogens in only 21 samples (11\%), five of those being parasites. Thus, the number of parasites not detectable by the multiplex approach is relatively high, most of which are not considered to be typical causes of gastroenteritis. On the other hand, 16 non-parasitic pathogens (9\%) would have been missed by microscopy alone. B. hominis and Endolimax nana are generally considered non-pathogenic, although they have been found in the stool of patients presenting with diarrhea. It is presently unresolved, whether these agents should be included in a multiplex panel. Our preliminary results on immunodeficient adult patients with diarrhea identified a higher detection rate of $29 \%$ with norovirus $(10 \%)$, C. difficile (4\%), and Campylobacter sp. (4\%). A similar distribution in immunodeficient patients with norovirus and Campylobacter sp. being the primary pathogens detected, has been observed by Coste et al. [10] and is emphasized in a recent review [12].

MultiplexNAT has a turnaround time (TAT) of approximately $6 \mathrm{~h}$, after which the results for 15 infectious agents are available in the electronic lab information system. By comparison, culture can take several days (at least $48 \mathrm{~h}$ ) and has a high hands-on-time. This is similar for microscopy, but typically multiple consecutive specimens are needed to diagnose a parasitic infection. Only DAD is faster than multiplexNAT, but the sensitivity, as shown in this study, is typically lower.

MultiplexNAT permits parallel testing of different pathogens and 20-40 patients are easily handled. Combined with automated extraction, we estimate the hands-ontime as being $2 \mathrm{~h}$ to generate 300 separate results for 20 patients. This approach also justifies higher costs (approx. $60 €$ of material per patient) of the multiplexNAT compared to conventional methods. DAD also has significant kit costs and shares a higher hands-on-time with microscopy. Clearly, the multiplexNAT requires the expertise of a molecular diagnostic laboratory with skilled personnel and is therefore not suitable as a point of care test. For urgent testing, other assays like DAD would be desirable.

For children with gastroenteritis, DAD is done first, and if found negative, or additional pathogens are clinically suspected, multiplexNAT is performed. For patients returning from the tropics, we would recommend to restrict multiplexNAT to patients with the clinical diagnosis of gastroenteritis. For immunocompromised patients or patients with a broad differential diagnosis, multiplexNAT could provide results rapidly that have an impact on the clinical management, e.g. infection control, cohorting, empiric therapy, resulting in reduced hospital stay and costs. In a second step, targeted tests can be added, e.g. antibiotic resistance, if needed.

Our study has some limitations: not all samples were tested by all methods. Because of the higher sensitivity of multiplexNAT, we selected positive samples and a random selection of 72 negative samples for independent confirmation. Second, we did not obtain positive results for all multiplexNAT targets. For example, the ability to detect $V$. cholerae or $Y$. enterocolitica could not be examined in our consecutive stool specimens. However, external quality control programs and other publications [10,27] strongly suggest a reasonable sensitivity. If these pathogens are suspected, we currently recommend using standard methods in addition to multiplexNAT.

Taken together, multiplexNAT can reduce the turnaround time and minimize hands-on-time and the need for splitting the specimens for parallel testing. Currently, we see the major role of multiplexNAT for patients with a broad differential diagnosis, especially when rapid results are needed. In fact, we reported a recent case of a hematopoietic stem cell transplant patient with multifactorial diarrhea, where the Shiga toxin-producing E. coli O51:H49 was first detected by multiplexNAT, which later developed a hemolytic uremic syndrome (HUS) with a fatal outcome [14]. To fully estimate the clinical importance of multiplexNAT, prospective studies are needed that address the role for diagnosis, infection control and empiric management of gastroenteritis patients.

Acknowledgments We thank Sibylle Stauffer, Hülya Atici, Rita Reuter, Michelle Dobler, Grethe Sägesser, and Nicole Cernela for excellent technical assistance, as well as Roger Stephan, and Herbert Hächler from the National Centre for Enteropathogenic Bacteria and Listeria (NENT) for testing and for helpful advice.

Conflict of interest The authors declare that they have no conflict of interest.

\section{References}

1. Barbut F, Corthier G, Charpak Y, et al. Prevalence and pathogenicity of Clostridium difficile in hospitalized patients. A French multicenter study. Arch Intern Med. 1996;156:1449-54.

2. Barnes GL, Uren E, Stevens KB, et al. Etiology of acute gastroenteritis in hospitalized children in Melbourne, Australia, from April 1980 to March 1993. J Clin Microbiol. 1998;36:133-8.

3. Bernier C, Gounon P, Le Bouguenec C. Identification of an aggregative adhesion fimbria (AAF) type III-encoding operon in enteroaggregative Escherichia coli as a sensitive probe for detecting the AAF-encoding operon family. Infect Immun. 2002;70:4302-11.

4. Black RE. Epidemiology of travelers' diarrhea and relative importance of various pathogens. Rev Infect Dis. 1990;12:S73-9.

5. Boisen N, Scheutz F, Rasko DA, et al. Genomic characterization of enteroaggregative Escherichia coli from children in Mali. J Infect Dis. 2012;205:431-44. 
6. Bon F, Fascia P, Dauvergne M, et al. Prevalence of group A rotavirus, human calicivirus, astrovirus, and adenovirus type 40 and 41 infections among children with acute gastroenteritis in Dijon, France. J Clin Microbiol. 1999;37:3055-8.

7. Calderaro A, Gorrini C, Montecchini S, et al. Evaluation of a realtime polymerase chain reaction assay for the laboratory diagnosis of giardiasis. Diagn Microbiol Infect Dis. 2010;66:261-7.

8. Casemore DP, Jackson B. Sporadic cryptosporidiosis in children. Lancet. 1983;2:679.

9. Cohen SH, Gerding DN, Johnson S, et al. Clinical practice guidelines for Clostridium difficile infection in adults: 2010 update by the society for healthcare epidemiology of America (SHEA) and the infectious diseases society of America (IDSA). Infect Control Hosp Epidemiol. 2010;31:431-55.

10. Coste JF, Vuiblet V, Moustapha B, et al. Microbiological diagnosis of severe diarrhea in kidney transplant recipients by use of multiplex PCR assays. J Clin Microbiol. 2013;51:1841-9.

11. Denno DM, Shaikh N, Stapp JR, et al. Diarrhea etiology in a pediatric emergency department: a case control study. Clin Infect Dis. 2012;55:897-904.

12. Dupont HL. Acute infectious diarrhea in immunocompetent adults. N Engl J Med. 2014;370:1532-40.

13. Farkas T, Jiang X, Guerrero ML, et al. Prevalence and genetic diversity of human caliciviruses (HuCVs) in Mexican children. J Med Virol. 2000;62:217-23.

14. Fasel D, Mellmann A, Cernela N, et al. Hemolytic uremic syndrome in a 65 year-old male linked to a very unusual type of stx2e and eae harboring O51:H49 Shiga-toxin producing Escherichia coli. J Clin Microbiol. 2014;52:1301-3.

15. Flem ET, Latipov R, Nurmatov ZS, et al. Costs of diarrheal disease and the cost-effectiveness of a rotavirus vaccination program in kyrgyzstan. J Infect Dis. 2009;200:S195-202.

16. Henriksen SA, Pohlenz JF. Staining of cryptosporidia by a modified Ziehl-Neelsen technique. Acta Vet Scand. 1981;22:594-6.

17. Jiang ZD, Lowe B, Verenkar MP, et al. Prevalence of enteric pathogens among international travelers with diarrhea acquired in Kenya (Mombasa), India (Goa), or Jamaica (Montego Bay). J Infect Dis. 2002;185:497-502.

18. Johnson S, Gerding DN. Clostridium difficile-associated diarrhea. Clin Infect Dis. 1998;26:1027-34 (quiz 1035-1026).

19. Kirkwood CD, Bishop RF. Molecular detection of human calicivirus in young children hospitalized with acute gastroenteritis in Melbourne, Australia, during 1999. J Clin Microbiol. 2001;39: 2722-4.

20. Laubach HE, Bentley CZ, Ginter EL, et al. A study of risk factors associated with the prevalence of Cryptosporidium in villages around Lake Atitlan, Guatemala. Braz J Infect Dis. 2004;8: 319-23.

21. Lindell AT, Grillner L, Svensson L, et al. Molecular epidemiology of norovirus infections in Stockholm, Sweden, during the years 2000 to 2003: association of the GGIIb genetic cluster with infection in children. J Clin Microbiol. 2005;43:1086-92.

22. Liu L, Johnson HL, Cousens S, et al. Global, regional, and national causes of child mortality: an updated systematic analysis for 2010 with time trends since 2000. Lancet. 2012;379:2151-61.
23. Marie-Cardine A, Gourlain K, Mouterde O, et al. Epidemiology of acute viral gastroenteritis in children hospitalized in Rouen, France. Clin Infect Dis. 2002;34:1170-8.

24. Marti H, Escher E. SAF-an alternative fixation solution for parasitological stool specimens. Schweiz Med Wochenschr. 1990;120:1473-6.

25. Martin-Ampudia M, Mariscal A, Lopez-Gigosos RM, et al. Under-notification of cryptosporidiosis by routine clinical and laboratory practices among non-hospitalised children with acute diarrhoea in Southern Spain. Infection. 2012;40:113-9.

26. Meinhardt PL, Casemore DP, Miller KB. Epidemiologic aspects of human cryptosporidiosis and the role of waterborne transmission. Epidemiol Rev. 1996;18:118-36.

27. Mengelle C, Mansuy JM, Prere MF, et al. Simultaneous detection of gastrointestinal pathogens with a multiplex Luminex-based molecular assay in stool samples from diarrhoeic patients. Clin Microbiol Infect. 2013;19:E458-65.

28. Moore JE, Madden RH. Survival of Campylobacter coli in porcine liver. Food microbiology. New York: Academic Press; 2001. p. $1-10$.

29. Morin N, Tirling C, Ivison SM, et al. Autoactivation of the AggR regulator of enteroaggregative Escherichia coli in vitro and in vivo. FEMS Immunol Med Microbiol. 2010;58:344-55.

30. Murray PR, Boron EJ, Jorgensen JH, Landry ML, Pfaller MA. Manual of clinical microbiology. Washington: American Society of Microbiology Press; 2007.

31. Nataro JP, Kaper JB. Diarrheagenic Escherichia coli. Clin Microbiol Rev. 1998;11:142-201.

32. Owen RJ, On SL, Costas M. The effect of cooling rate, freezedrying suspending fluid and culture age on the preservation of Campylobacter pylori. J Appl Bacteriol. 1989;66:331-7.

33. Rheingans R, Kukla M, Adegbola RA, et al. Exploring household economic impacts of childhood diarrheal illnesses in three African settings. Clin Infect Dis. 2012;55:S317-26.

34. Roman E, Wilhelmi I, Colomina J, et al. Acute viral gastroenteritis: proportion and clinical relevance of multiple infections in Spanish children. J Med Microbiol. 2003;52:435-40.

35. Scheier E, Aviner S. Septicemia following rotavirus gastroenteritis. Isr Med Assoc J. 2013;15:166-9.

36. Schmidt H, Knop C, Franke S, et al. Development of PCR for screening of enteroaggregative Escherichia coli. J Clin Microbiol. 1995;33:701-5.

37. Shkalim V, Amir A, Samra Z, et al. Characteristics of non-typhi Salmonella gastroenteritis associated with bacteremia in infants and young children. Infection. 2012;40:285-9.

38. Simpson R, Aliyu S, Iturriza-Gomara M, et al. Infantile viral gastroenteritis: on the way to closing the diagnostic gap. J Med Virol. 2003;70:258-62.

39. Stark D, Al-Qassab SE, Barratt JL, et al. Evaluation of multiplex tandem real-time PCR for detection of Cryptosporidium spp., Dientamoeba fragilis, Entamoeba histolytica, and Giardia intestinalis in clinical stool samples. J Clin Microbiol. 2011;49: 257-62.

40. Thielman NM, Guerrant RL. Clinical practice. Acute infectious diarrhea. N Engl J Med. 2004;350:38-47. 